\title{
An improved method using factor division algorithm for reducing the order of linear dynamical system
}

\author{
SHARAD KUMAR TIWARI* and GAGANDEEP KAUR \\ Department of Electrical and Instrumentation Engineering, Thapar University, Patiala 147004, India \\ e-mail: sktiwari@thapar.edu; gagandeep@thapar.edu
}

MS received 2 August 2014; revised 4 December 2015; accepted 13 February 2016

\begin{abstract}
An improved method is proposed to determine the reduced order model of large scale linear time invariant system. The dominant poles of the low order system are calculated by clustering method. The selection of pole to the cluster point is based on the contributions of each pole in redefining time moment and redefining Markov parameters. The coefficients of the numerator polynomial for reduced model are obtained using a factor division algorithm. This method is computationally efficient and keeps up the stability and input output characteristic of the original arrangement.
\end{abstract}

Keywords. Model order reduction; integral square error; linear time invariant; factor division algorithm; time moment and Markov parameter.

\section{Introduction}

Modelling of higher order dynamical systems is hard to handle because of computation and implementation difficulties, and they are too complex to be used in real time problem. Model order reduction (MOR) is a technique to simplify the high order dynamical systems described by differential equations. The intention is to supplant the high dimensional system into a low-dimensional system by preserving the attributes of the original arrangement. Several methods are available in the literature for reducing the linear time invariant system in time and frequency domain. The stability based method preserves stability in the reduced model, if the original system is stable [1]. Parthasarathy and Jayasimha [2] combined the method with modified Cauer continued fraction technique in order to hold the social status of the original system in the reduced order model. The differentiation based reduction method was introduced by Gautam et al [3]. The reciprocal of numerator and denominator polynomial of a high order transfer function is differentiated many times to generate the coefficients of the reduced transfer function $[4,5]$. The drawback of this method is that steady state does not always match. In the clustering method suggested by Sinha and Pal [6], the poles and zeroes are individually grouped to form clusters and then these clusters are replaced by their cluster centers by using an inverse distance measure (IDM) criterion [7-9]. Only the poles are grouped together to generate cluster centers and then denominator polynomial of the reduced model is formed from these cluster centers, but

*For correspondence difficulty with these methods is the selection of poles for cluster point. To avert this problem an improved method is introduced using redefine Markov parameter and time moment [10]. This progresses to the selection of poles. The factor division algorithm $[4,11]$ can be used to determine the numerator polynomial of the reduced model. The factor division algorithm provides a good reduced order approximation of original high order systems since it avoids the finding of time moments and solving the Pade equation, while retain the initial time moments of the original systems.

In this proposed algorithm for order reduces the trouble of selecting poles is removed by applying the advantages of time moment and Markov parameters which are specified as a subroutine of the poles and residues of the transport function of the original model having real and complex poles. Here poles from the original model are selected by their highest contribution in redefined time moment (RTMs) and lowest contributions in redefining Markov parameters (RMPs). The selected poles are used to get the cluster center using the inverse distance method to get dominant pole. The coefficients of the numerator polynomial of reduced model are obtained using a factor division algorithm.

\section{Problem formulation}

Let us consider the input output relationship of a linear time invariant system defined by the following differential equation 


$$
\begin{aligned}
& b_{n} \frac{\mathrm{d}^{n} y(t)}{\mathrm{d} t^{n}}+b_{n-1} \frac{\mathrm{d}^{n-1} y(t)}{\mathrm{d} t^{n-1}}+\cdots+b_{1} \frac{\mathrm{d} y(t)}{\mathrm{d} t}+b_{0} y(t) \\
& \quad=a_{m} \frac{\mathrm{d}^{m} u(t)}{\mathrm{d} t^{m}}+a_{m-1} \frac{\mathrm{d}^{m-1} u(t)}{\mathrm{d} t^{m-1}}+\cdots+a_{1} \frac{\mathrm{d} u(t)}{\mathrm{d} t}+a_{0} u(t)
\end{aligned}
$$

where $y$ is the output of the system and $u$ is the input. Equation (1) can be written in the course of the transfer function as

$$
G(s)=\frac{a_{m} s^{m}+a_{m-1} s^{m-1}+\cdots+a_{1} s+a_{0}}{b_{n} s^{n}+b_{n-1} s^{n-1}+\cdots+b_{1} s+b_{0}}
$$

where $m<n, D(s)=\prod_{i=1}^{n}\left(s-\lambda_{i}\right) ; n$ is the number of poles and $N(s)=\prod_{i=1}^{m}\left(s-\delta_{i}\right) ; m$ is the number of zeros, $\lambda_{i}=\left(\alpha_{i} \pm j \beta_{i}\right)$ and $\delta_{i}=\left(\varepsilon_{i}+j \gamma_{i}\right)$ of the original high order system $G_{n}(s)$. The poles and zero may be either real or complex or both combination of real and complex. If the poles are complex they occur in conjugate pair.

The corresponding reduced $r$ th order is given by

$$
G_{r}(s)=\frac{N_{r-1}(s)}{D_{r}(s)}=\frac{c_{r-1} s^{r-1}+c_{r-2} s^{r-2}+\cdots+c_{1} s+c_{0}}{d_{r} s^{r}+d_{r-1} s^{r-1}+\cdots+d_{1} s+d_{0}}
$$

where $D_{r}(s)=\prod_{i=1}^{r}\left(s-\lambda_{i}^{\prime}\right) ; r$ is the number of poles and $\alpha_{i j}(s)=\prod_{i=1}^{r-1}\left(s-\delta_{i}^{\prime}\right) ; m$ is the number of zeros, $\lambda_{i}^{\prime}=$ $\left(\alpha_{i}^{\prime} \pm j \beta_{i}^{\prime}\right)$ and $\delta_{i}=\left(\varepsilon_{i}^{\prime}+j \gamma_{i}^{\prime}\right)$ of the reduced order model $G_{r}(s)$. The poles and zero may be either real or complex or both combination of real and complex. The objective of the model order reduction is to reduce the order of a given linear system, for maintaining the system stability, produce response with minimum error.

\section{Description of method}

Step 1: Determination of $r$ th order denominator polynomial First dominant pole calculation

The denominator polynomial of original $n$ th-order transfer function is given as

$$
D_{n}(s)=b_{n} s^{n}+b_{n-1} s^{n-1}+\cdots+b_{1} s+b_{0}=\prod_{i=1}^{n}\left(s-\lambda_{i}\right) .
$$

Let there be $n$ poles $\left|\lambda_{1}\right|<\left|\lambda_{2}\right|<\left|\lambda_{3}\right|<\cdots<\left|\lambda_{n}\right|$. The approximate value of one dominant pole can be calculated by

$$
\lambda_{1}^{\prime}=\sqrt{1-\frac{\lambda_{1}}{\lambda_{n}}} .
$$

The next approximate $r-1$ dominant poles

We know that the power series expansion of $G(s)$ about $s=0$ is

$$
G(s)=C_{0}+C_{1} s+C_{2} s^{2}+\cdots=\sum_{i=0}^{\infty} C_{i} s^{i}
$$

where $C_{i}=\frac{(-1)^{i}}{i !} \mathrm{RTM}_{i} ; i=0,1,2,3, \ldots$

The expansion of $G(s)$ about $s=\infty$ is

$$
\begin{aligned}
& G(s)=\mu_{0} s^{-(n-m)}+\mu_{1} s^{-(n-m+1)}+\mu_{2} s^{-(n-m+2)}+\cdots \\
& G(s)=\sum_{i=0}^{\infty} \mu_{i} s^{-(n-m+i)}
\end{aligned}
$$

where $\mu_{i}=\mathrm{RMP}_{i} ; i=0,1,2,3, \ldots$.

Defined the expression for redefined time moments (RTMs) as

$$
\mathrm{RTM}_{i}=\sum_{j=1}^{n} x_{i j}
$$

where $x_{i j}=i ! \frac{P_{j}}{\lambda_{j}^{(i+1)}} ; i=0,1,2, \ldots$

Defined the expression for redefined Markov parameters (RMPs) as

$$
\mathrm{RMP}_{i}=\sum_{j=1}^{n} y_{i j}
$$

where $y_{i j}=P_{j} \lambda_{j}^{i} ; i=0,1,2,3, \ldots$ and $P_{j}$ are residues.

The reduced denominator polynomial $D_{r}(s)$ is obtained by taking one pole from the dominant pole calculation using Eq. (5), which is close to the origin. Remaining poles of the reduced model are obtained by taking cluster centre using inverse distance method. Clusters are formed by the poles having highest contributions in RTMs and poles having lowest contributions in RMPs according to their contribution weighted respectively as indicated in table 1 . These selected poles give $r-1$ cluster point using Eq. (10).

$$
\lambda_{i}^{\prime}=\left[\sum_{j=1}^{k}\left(\frac{-1}{\left|p_{j}\right|}\right) \div k\right]^{-1} .
$$

where $j=1,2, \ldots, k$.

$k$ is the number of poles selected from RTMs or RMPs to get cluster centre. If poles are complex conjugate $\left[\left(\vartheta_{1} \pm w_{1}\right),\left(\vartheta_{2} \pm w_{2}\right), \ldots,\left(\vartheta_{i} \pm w_{i}\right)\right]$ then the inverse distance measure criterion finds the complex cluster centre in the form of $A_{c} \pm B_{c} . A_{c}=\left\{\left[\sum_{i=1}^{m}\left(1 / \vartheta_{i}\right) / 2\right]\right\}^{-1}$ and $B_{c}=\left\{\left[\sum_{i=1}^{m}\left(1 / w_{i}\right) / 2\right]\right\}^{-1}$.

Table 1. Contribution of individual poles.

\begin{tabular}{lcccccccc}
\hline Parameter & $\lambda_{1}$ & $\lambda_{2}$ & $\lambda_{3}$ & $\ldots$ & $\lambda_{\mathrm{j}}$ & $\ldots$ & $\lambda_{\mathrm{n}}$ & Sum \\
\hline RTMs & $x_{\mathrm{i} 1}$ & $x_{\mathrm{i} 1}$ & $x_{\mathrm{i} 1}$ & $\ldots$ & $x_{\mathrm{ij}}$ & $\ldots$ & $x_{\mathrm{in}}$ & $\mathrm{RTM}_{\mathrm{i}}$ \\
RMPs & $y_{\mathrm{i} 1}$ & $y_{\mathrm{i} 1}$ & $y_{\mathrm{i} 1}$ & $\cdots$ & $y_{\mathrm{ij}}$ & $\cdots$ & $y_{\mathrm{in}}$ & $\mathrm{RMP}_{\mathrm{i}}$ \\
\hline
\end{tabular}


Step 2: Determination of the numerator polynomial of $(r-1)$ th order using factor division algorithm [12], $N_{r-1}(s)$ in Eq. (3) can be given by

$$
N_{r-1}(s)=\frac{N(s)}{D(s)} \times D_{r}(s),
$$

where $D_{r}(s)$ are obtained. Therefore, the numerator $N_{r}(s)$ of the reduced order model $G_{r}(s)$ in Eq. (2) will be the series expansion of

$$
\begin{aligned}
G_{r}(s) & =\frac{N(s)}{D(s) / D_{r}(s)}=\frac{\sum_{i=0}^{n-1} b_{i} s^{i}}{\sum_{i=0}^{n-r} e_{i} s^{i}}, \text { about } s \\
& =0 \text { up to the term of orders }{ }^{r-1} .
\end{aligned}
$$

This is calculated by a modified form of a moment generating algorithm, which uses the Routh recurrence formula to give the third, fifth, seventh, etc., rows as

$$
\begin{aligned}
& c_{0}=\frac{b_{0}}{e_{0}}\left\langle\begin{array}{ccccc}
b_{0} & b_{1} & b_{2} & \ldots & b_{r-1} \\
e_{0} & e_{1} & e_{2} & \ldots & e_{r-1}
\end{array}\right. \\
& c_{1}=\frac{q_{0}}{e_{0}}\left\langle\begin{array}{llll}
q_{0} & q_{1} & \ldots & q_{r-2} \\
e_{0} & e_{1} & \ldots & e_{r-2}
\end{array}\right. \\
& c_{2}=\frac{r_{0}}{e_{0}}\left\langle\begin{array}{lll}
r_{0} & \cdots & r_{r-3} \\
e_{0} & \cdots & e_{r-3}
\end{array}\right. \\
& c_{r-2}=\frac{u_{0}}{e_{0}}\left\langle\begin{array}{lll}
u_{0} & \cdots & u_{r-4} \\
e_{0} & \cdots & e_{r-4}
\end{array}\right. \\
& c_{r-2}=\frac{v_{0}}{e_{0}}\left\langle\begin{array}{l}
v_{0} \\
e_{0}
\end{array}\right.
\end{aligned}
$$

\section{Numerical experiments}

\section{Numerical example: 1}

Consider the eighth-order linear dynamic system described by the transfer function [13].

$$
G_{8}(s)=\frac{18 s^{7}+514 s^{6}+5982 s^{5}+36380 s^{4}+122664 s^{3}+185760 s^{2}+185760 s+40320}{s^{8}+36 s^{7}+546 s^{6}+4536 s^{5}+22449 s^{4}+67284 s^{3}+118124 s^{2}+109584 s+40320}
$$

where

$$
\begin{aligned}
& q_{i}=b_{i+1}-c_{0} e_{i+1}, i=0,1, \ldots, r-2 \\
& r_{i}=q_{i+1}-c_{1} e_{i+1}, i=0,1, \ldots, r-3 \\
& \vdots \ldots \ldots \\
& v_{0}=u_{1}-c_{r-2} e_{1} .
\end{aligned}
$$

Therefore the numerator of the reduced order model is obtained as
The poles of the system are $\lambda_{1}=-1, \lambda_{2}=-2$, $\lambda_{3}=-3, \lambda_{4}=-4, \lambda_{5}=-5, \lambda_{6}=-6, \lambda_{7}=-7$ and $\lambda_{8}=-8$. The first dominant pole is $\lambda_{1}^{\prime}=-0.9922$ using Eq. (5) and second dominant pole is calculated by using Eq. (10). The poles are selected for clustering based on the contributions of individual poles in RMPs from table 2. The poles having lowest contributions in RMPs are $\lambda_{8}, \lambda_{7}$ and $\lambda_{4}$. Thus, from Eq. (10) we get $\lambda_{2}^{\prime}=-5.7931$.

The transfer function of the reduced second order system using the proposed method is 
Table 2. Contribution of individual poles of example 1.

\begin{tabular}{lccccccrrr}
\hline Parameters & $\lambda_{1}$ & $\lambda_{2}$ & $\lambda_{3}$ & $\lambda_{4}$ & $\lambda_{5}$ & \multicolumn{1}{c}{$\lambda_{6}$} & \multicolumn{1}{c}{$\lambda_{7}$} & \multicolumn{1}{c}{$\lambda_{8}$} \\
\hline RTM0 & -3.0 & 2.02 & -2.23 & 2.89 & -0.74 & -0.20 & 1.05 & 1.20 \\
RTM1 & -3.0 & 1.01 & -0.74 & 0.72 & -0.15 & -0.03 & 0.15 & 0.15 \\
RMP0 & -3.0 & 4.04 & -6.68 & 11.56 & -3.68 & -1.20 & 7.33 & 9.63 & 1.00 \\
RMP1 & 3.004 & -8.088 & 20.025 & -46.22 & 18.4 & 7.2 & -51.31 & -77 & -133.99 \\
\hline
\end{tabular}

Table 3. Quantitative analysis with the original system.

\begin{tabular}{lcr}
\hline & 8th order original system & 2nd order reduced system \\
\hline Settling time (s) & 4.82 & 4.57 \\
Rise time (s) & 0.0647 & 0.0608 \\
Overshoot (\%) & 120 & 123 \\
\hline
\end{tabular}

Table 4. Comparison of model order reduction methods.

\begin{tabular}{lcc}
\hline Reduction method & 2nd order reduced model & ISE \\
\hline Proposed method & $G_{2}(s)=\frac{17.74 s+5.793}{s^{2}+6.793 s+5.793}$ & 0.00544 \\
Vishwakarma [13] & $G_{2}(s)=\frac{16.511145 s+5.45971}{s^{2}+6.19642 s+5.45971}$ & 0.01406 \\
Parmar et al [11] & $G_{2}(s)=\frac{24.11429 s+8}{s^{2}+9 s+8}$ & 0.04809 \\
Mukherjee and Satakshi [14] & $G_{2}(s)=\frac{11.3909 s+4.4357}{s^{2}+4.2122 s+4.4357}$ \\
Mittal et al [15] & $G_{2}(s)=\frac{7.0908 s+1.9906}{s^{2}+3 s+2}$ & 0.056897 \\
Prasad and Pal [16] & $G_{2}(s)=\frac{17.98561 s+500}{s^{2}+13.24571 s+500}$ \\
\hline
\end{tabular}

$$
G_{2}(s)=\frac{17.04 s+5.748}{s^{2}+6.785 s+5.748}
$$

The comparison of transient response specification is depicted in table 3 . The proposed method is compared with the other order-reduction method available in literature and are shown in table 4 for example 1 by comparing the integral square error in between the step responses of the original $G_{n}(\mathrm{~s})$ and reduced $G_{r}(s)$ order systems. Figure 1 shows the comparison with step responses of original model and reduced model of example 1. Similarly, the frequency responses are compared as displayed in figure 2.

\section{Example: 2}

Consider the sixth-order multivariable linear dynamic system described by the transfer matrix [9]

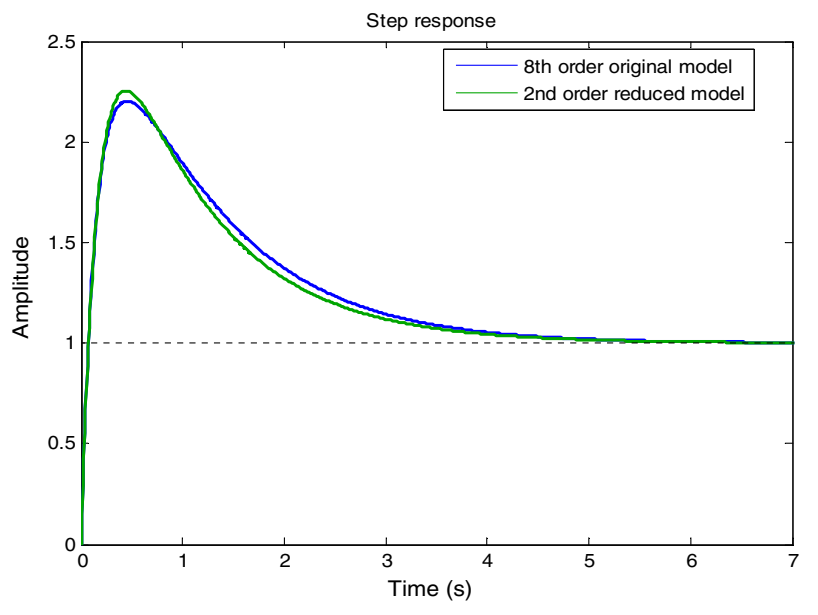

Figure 1. Comparison with step responses of original model and reduced model of example 1. 


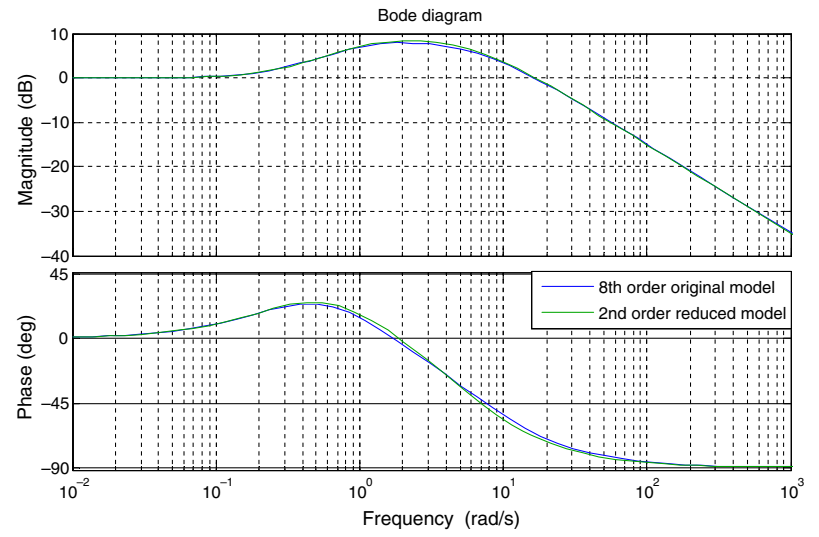

Figure 2. Comparison of frequency responses of example 1.

$$
[G(s)]=\left(\begin{array}{cc}
\frac{2(s+5)}{(s+1)(s+10)} & \frac{(s+4)}{(s+2)(s+5)} \\
\frac{(s+10)}{(s+1)(s+20)} & \frac{(s+6)}{(s+3)(s+3)}
\end{array}\right)
$$

The minimal denominator polynomial is of order six and is given by

$$
\begin{aligned}
D_{6}(s)= & (s+1)(s+2)(s+3)(s+5)(s+10)(s+20) \\
= & s^{6}+41 s^{5}+571 s^{4}+3491 s^{3} \\
& +10060 s^{2}+13100 s+6000 .
\end{aligned}
$$

Let

$$
[G(s)]=\frac{1}{D_{6}(s)}\left(\begin{array}{ll}
a_{11}(s) & a_{12}(s) \\
a_{21}(s) & a_{22}(s)
\end{array}\right)
$$

$$
\begin{aligned}
& a_{11}(s)=2 s^{5}+70 s^{4}+762 s^{3}+3610 s^{2}+7000 s+6000 \\
& a_{12}(s)=s^{5}+38 s^{4}+459 s^{3}+2182 s^{2}+4160 s+2400 \\
& a_{21}(s)=s^{5}+30 s^{4}+331 s^{3}+1650 s^{2}+3700 s+3000 \\
& a_{22}(s)=s^{5}+42 s^{4}+601 s^{3}+3600 s^{2}+9100 s+6000
\end{aligned}
$$

The contribution of individual poles of RTMs and RMPs are presented in table 5. The corresponding transfer matrix of second order reduced model is

$$
\begin{aligned}
{\left[G_{2}(s)\right]=} & \frac{1}{s^{2}+2.818 s+1.818} \\
& \times\left(\begin{array}{cc}
1.097 s+1.8183 & 0.8003 s+0.7273 \\
0.545 s+0.9092 & 1.605 s+1.8183
\end{array}\right) .
\end{aligned}
$$

\begin{tabular}{|c|c|c|c|c|c|c|c|c|c|c|c|c|c|c|c|c|}
\hline \multirow[b]{2}{*}{ Parameters } & \multicolumn{7}{|c|}{$a_{11}(s)$} & \multicolumn{9}{|c|}{$a_{12}(s)$} \\
\hline & $\lambda_{1}$ & $\lambda_{2}$ & $\lambda_{3}$ & & $\lambda_{4}$ & $\lambda_{5}$ & $\lambda_{6}$ & Sum & $\lambda_{1}$ & $\lambda_{2}$ & $\lambda_{3}$ & & & $\lambda_{5}$ & $\lambda_{6}$ & Sum \\
\hline $\mathrm{RTM}_{0}$ & 0.89 & 0 & 0 & & 0 & 0.11 & 0 & 1 & 0 & 0.33 & 0 & & & 0 & 0 & 0.4 \\
\hline $\mathrm{RTM}_{1}$ & 0.89 & 0 & 0 & & 0 & 0.01 & 0 & 0.9 & 0 & 0.17 & 0 & & & 0 & 0 & 0.18 \\
\hline $\mathrm{RMP}_{0}$ & 0.89 & 0 & 0 & & 0 & 1.11 & 0 & 2 & 0 & 0.67 & 0 & & & 0 & 0 & 1 \\
\hline \multirow[t]{2}{*}{$\mathrm{RMP}_{1}$} & -0.89 & 0 & 0 & & 0 & -11.11 & 0 & -12 & 0 & -1.33 & 0 & & & 0 & 0 & -3 \\
\hline & \multicolumn{7}{|c|}{$a_{21}(s)$} & \multicolumn{9}{|c|}{$a_{22}(s)$} \\
\hline $\mathrm{RTM}_{0}$ & 0.47 & 0 & 0 & 0 & 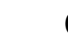 & 0 & & 0.5 & 0 & 2.0 & & & 0 & 0 & 0 & 1 \\
\hline $\mathrm{RTM}_{1}$ & 0.47 & 0 & 0 & 0 & 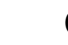 & 0 & & 0.475 & 0 & 1.0 & & & 0 & 0 & 0 & 0.7 \\
\hline $\mathrm{RMP}_{0}$ & 0.47 & 0 & 0 & 0 & , & 0 & & 1 & 0 & 4.0 & & & 0 & 0 & 0 & 1 \\
\hline $\mathrm{RMP}_{1}$ & -0.47 & 0 & 0 & 0 & 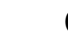 & -10 & & -11 & 0 & -8.0 & & 9.0 & 0 & 0 & 0 & 1 \\
\hline
\end{tabular}

Transient response specifications of original and reduced

\begin{tabular}{|c|c|c|c|c|c|c|c|c|}
\hline & \multicolumn{4}{|c|}{ 6th order original system } & \multicolumn{4}{|c|}{2 nd order reduced model } \\
\hline & $G_{11}(s)$ & $G_{12}(s)$ & $G_{21}(s)$ & $G_{22}(s)$ & $G_{11}(s)$ & $G_{12}(s)$ & $G_{21}(s)$ & $G_{22}(s)$ \\
\hline Settling time (s) & 3.79 & 3.86 & 1.87 & 2.3 & 3.88 & 3.8 & 1.62 & 2.82 \\
\hline Rise time (s) & 2.13 & 2.18 & 1.01 & 1.34 & 2.11 & 2.11 & 1.02 & 1.48 \\
\hline Overshoot $(\%)$ & 0 & 0 & 0 & 0 & 0 & 0 & 0 & 0 \\
\hline
\end{tabular}
model are compared in table 6. The proposed method is compared with the other order-reduction methods available in literature and are shown in table 7 for example 3 by comparing the integral square error in between the step responses of the original $\left[G_{n}(s)\right]$ and reduced $\left[G_{r}(s)\right]$ order systems. Figure 3 shows the comparison with step responses of original model and reduced model of example 2 .

Table 5. Contribution of poles in RTMs and RMPs in $G_{n}(s)$.

Table 6. Quantitative analysis with the original system. 
Table 7. Comparison of model order reduction methods.

\begin{tabular}{|c|c|c|c|c|}
\hline \multirow[b]{2}{*}{ Reduction methods } & \multicolumn{4}{|c|}{ ISE for $G_{\mathrm{ij}}(s)$} \\
\hline & $G_{11}(s)$ & $G_{12}(s)$ & $G_{21}(s)$ & $G_{22}(s)$ \\
\hline Proposed method & 0.001095 & 0.00021 & 0.000081 & 0.002800 \\
\hline Vishwakarma and Prasad [9] & 0.001515 & $7.845 \times 10^{-5}$ & 0.000299 & 0.004681 \\
\hline Parmar et al [11] & 0.014498 & 0.008744 & 0.002538 & 0.015741 \\
\hline Prasad et al [5] & 0.136484 & 0.002446 & 0.040291 & 0.067902 \\
\hline Safonoy and Chiang [17] & 0.590617 & 0.037129 & 0.04291 & 1.066123 \\
\hline
\end{tabular}

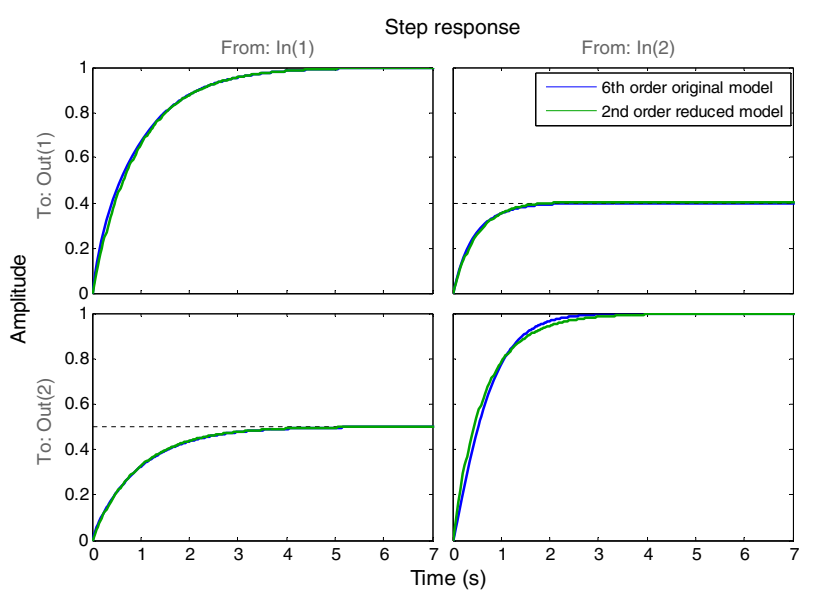

Figure 3. Comparison with step responses of original model and reduced model of example 2 .

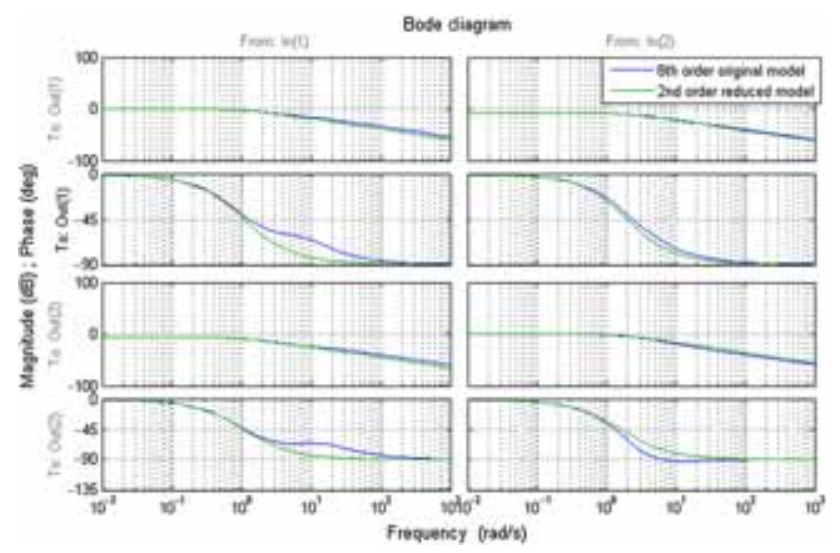

Figure 4. Comparison of frequency responses of example 2.

Similarly, the frequency responses are compared as displayed in figure 4.

\section{Conclusion}

In this paper an improved mixed method is applied to set the reduced order model from large scale linear dynamic system. The method is to get the approximate dominant poles by using the advantages of the contributions of pole RTMs and RMPs for selecting the poles for clusters. The clusters give dominant pole for reduced model. Hence, the reduced order model estimates the approximation in almost entire frequency range. The coefficient of the numerator polynomial is obtained by factor division algorithms. This mixed method preserves the stability of the original system and produce a good approximation. The performance of the proposed method is analyzed by ISE criterion and compared with the other methods. This method has been extended to multivariable system.

\section{References}

[1] Chen T C, Chang T Y and Han K W 1979 Reduction of transfer functions by the stability equation method. $J$. Franklin Inst. 308(4): 389-404

[2] Parthasarathy R and Jayasimha K N 1982 System reduction using stability equation method and modified Cauer continued fraction. Proc. IEEE 70(10): 1234-1236

[3] Gautam P O, Mannerfelt C F and Monlander 1992 Contribution to the model reduction problem. IEEE Trans. Autom. Control 27(2): 454-455

[4] Lucas T N 1992 Some other observation on the differentiation method of model reduction. IEEE Trans. Autom. Control 37(9): 1389-1391

[5] Prasad R, Pal J and Pant A K 1995 Multivariable system approximation using polynomial derivatives. J. Inst. Eng. 76: 186-188

[6] Sinha A K and Pal J 1990 Simulation based reduced order modeling using a clustering technique. Comput. Electr. Eng. 16(3): 159-169

[7] Pal J, Sinha A K and Sinha N K 1995 Reduced-order modelling using pole clustering and time-moments matching. $J$. Inst. Eng. (India), Electr. Eng. 76: 1-6

[8] Vishwakarma C B and Prasad R 2008 Clustering methods for reducing order of linear system using Pade approximation. IETE J. Res. 54(5): 326-330

[9] Vishwakarma C B and Prasad R 2009 MIMO system reduction using modified pole clustering and genetic algorithm. Hindawi Publishing Corporation Modeling and Simulation in Engineering DOI:10.1155/2009/540895

[10] Saraswathi G 2011 An extended method for order reduction of large scale multivariable systems. Int. J. Comput. Sci. Eng. 2(6): 2109-2118 
[11] Parmar G, Mukherjee S and Prasad R 2007 System reduction using factor division algorithm and eigen spectrum analysis. Appl. Math. Modell. 31(11): 2542-2552

[12] Lucas T N 1980 Factor division: A useful algorithm in model reduction. Proceeding IEE 130(6): 362-364

[13] Vishwakarma C B 2011 Order reduction using modified pole clustering and Pade approximations. World Acad. Sci. Eng. Tech. 56: 787-791

[14] Mukherjee S, Satakshi and Mittal R C 2005 Model order reduction using response matching. J. Franklin Inst. 342: 503-519
[15] Mittal A K, Prasad R and Sharma S P 2004 Reduction of linear dynamic systems using an error minimization technique. J. Inst. Eng. IE(I) J. -EL 84: 201-206

[16] Prasad R and Pal J 1991 Stable reduction of linear systems by continued fractions. J. Inst. Eng. IE(I) J. 72: 113-116

[17] Safonoy M G and Chiang R Y 1988 Model reduction for robust control: A Schur relative error method. Proc. Int. J. Adapt. Control Signal 2(4): 259-272 